\title{
Co-morbidities of mental disorders and chronic physical diseases in developing and emerging countries: a meta-analysis
}

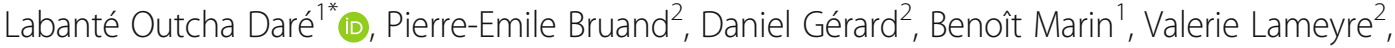 \\ Farid Boumédiène ${ }^{1}$ and Pierre-Marie Preux ${ }^{1}$
}

\begin{abstract}
Background: As the data on the association of mental disorders and chronic physical diseases in developing and emerging countries is heterogeneous, this study aims to produce the first meta-analysis of these comorbidities.

Methodology: The meta-analysis protocol was registered in PROSPERO ( $\left.{ }^{\circ} \mathrm{CRD} 42017056521\right)$ and was performed in accordance with PRISMA guidelines. Initially, an article search was conducted on Medline, Embase, Lilacs and the Institut d'Epidémiologie et de Neurologie Tropicale database [Institute of Epidemiology and Tropical Neurology], as well as manually, with no restriction on language or date focusing on mental disorders, chronic diseases and neurotropic diseases. Two independent investigators assessed the quality of the studies which met the inclusion criteria using the Downs and Black assessment grid. The pooled estimates were calculated out using a randomeffects method with CMA software Version 3.0. A meta-regression was then performed, and the significance level was set at 0.05 .

Results: Of the 2604 articles identified, 40 articles involving 21,747 subjects met the inclusion criteria for co-morbidities between mental disorders and chronic physical diseases. Thirty-one articles were included in the meta-analysis of prevalence studies and 9 articles in that of the analytical studies. The pooled prevalence of mental disorders in patients with chronic physical diseases was $36.6 \%$ (95\% Cl, 31.4-42.1) and the pooled odds ratio was $3.1(95 \% \mathrm{Cl}, 1.7-5.2)$. There was heterogeneity in all the estimates and in some cases, this was explained by the quality of the studies.

Conclusion: Some estimates regarding the prevalence of mental disorders in people with chronic physical diseases living in developing and emerging countries were similar to those in developed countries. Mental disorders are a burden in these countries. In order to respond effectively and efficiently to the morbidity and mortality associated with them, mental health care could be integrated with physical care.
\end{abstract}

Keywords: Meta-analysis, Association, Comorbidities, Mental disorders, Chronic diseases

\section{Background}

Worldwide, the burden of chronic physical diseases and mental disorders is becoming ever more significant $[1,2]$. This increase is largely attributable to the current epidemiological transition in many developing and emerging countries with an ongoing increase in non-communicable diseases in adults [3].

\footnotetext{
* Correspondence: outcha_dare1986@yahoo.com

${ }^{1}$ INSERM, Univ. Limoges, CHU Limoges, UMR_S 1094, Tropical

Neuroepidemiology, Institute of Neuroepidemiology and Tropical Neurology, CNRS FR 3503 GEIST, F-87000 Limoges, France

Full list of author information is available at the end of the article
}

Mental disorders, an essential component of these diseases, are a burden with a huge impact in terms of disability. In 2010, mental disorders and drug addiction accounted for 183.9 million Disability-Adjusted Life Years (DALYs), i.e., 7.4\% (6.2-8.6) of all DALYs worldwide [4]. However, the most recent data published by Vigo et al. in 2016 [5], suggest that the global burden of mental illness would actually account for $32.4 \%$ of Years Lived with Disability (YLDs), and for $13.0 \%$ of DALYs. In this crucial period of epidemiological transition, when developing and emerging countries are facing new health challenges, the studying of co-morbidities for chronic

(c) The Author(s). 2019 Open Access This article is distributed under the terms of the Creative Commons Attribution 4.0 International License (http://creativecommons.org/licenses/by/4.0/), which permits unrestricted use, distribution, and reproduction in any medium, provided you give appropriate credit to the original author(s) and the source, provide a link to the Creative Commons license, and indicate if changes were made. The Creative Commons Public Domain Dedication waiver (http://creativecommons.org/publicdomain/zero/1.0/) applies to the data made available in this article, unless otherwise stated. 
physical diseases is relevant because they represent the greatest cause of death in the world [6]. Mental disorders rank third among the most frequent diseases after cancer and cardiovascular diseases [7]. According to surveys conducted in developed and developing countries, more than $25.0 \%$ of individuals have one or more mental or behavioural disorders during their lifetime [8]. In the general population, severe characterized depression affects $3.0 \%$ of people, generalized anxiety disorder affects $2.0 \%$ and the prevalence of schizophrenia is expressed at nearly $1.0 \%$ [9]. In terms of chronic physical diseases, the world's leading causes of death, it was estimated in 2008 that 36 million deaths were due to chronic physical diseases [10], including $30.0 \%$ to cardiovascular diseases, $13.0 \%$ to cancers, $7.0 \%$ to chronic respiratory diseases, $2.0 \%$ to diabetes and $9.0 \%$ to other chronic diseases $[11,12]$.

This situation is even more worrying as mental disorders are often associated with one or more chronic physical diseases and lead to even more aggravating physical consequences for patient health. In addition, the care provided to people with mental disorders for their physical health problems is inferior to that received by persons without mental disorders. The figures indicate that in low- and middle-income countries approximately $80 \%$ of people with severe mental disorders have a problem accessing mental health care and do not receive treatment; this makes mental disorders the second highest cause of morbidity and mortality in these countries. The association between mental disorders (depression, anxiety, schizophrenia and bipolar disorders) and chronic physical diseases [13] such as cancer [14, 15], heart disease, stroke $[15,16]$, diabetes [17-20]), obesity [21] and chronic obstructive pulmonary disease (COPD) [22, 23] has been the subject of several studies. The data on these comorbidities is very heterogeneous in developing and emerging countries (all non-high income countries by World Bank rankings [24]). For some of these comorbidities, the prevalence rates range from 19.0 [25] to $68.1 \%$ [26] and some authors assert that these comorbidities are underestimated $[27,28]$ in these countries. There is, to date, no meta-analysis covering all the developing and emerging countries, and as such, a study might have a significant impact on public health guidelines for these countries; hence the reason why we decided to perform a meta-analysis of the association of mental disorders and chronic physical diseases.

\section{Purpose of the study}

The purpose of this study was to estimate the pooled prevalences and pooled association measures of thevarious associations of mental disorders and chronic physical diseases in developing and emerging countries.

\section{Methods}

\section{Implementation procedure}

When performing this meta-analysis, the diseases of interest were: - for mental disorders: anxiety, depression, bipolar disorder and schizophrenia [5, 29]. Mental health disorders are medical conditions that can interfere with thinking, feeling, mood, a person's ability to communicate with others and daily functioning. They are medical conditions that often result in a reduced ability to cope with routine daily activities: such as going to work or raising a family [13]. - for chronic physical diseases: diabetes, obesity, cancer, heart disease and COPD $[6,30]$. Chronic diseases are non-communicable diseases, prolonged in time, which do not resolve spontaneously, and which are rarely completely curable [5].

The meta-analysis was performed according to Preferred Reporting Items for Systematic Review and Meta-Analysis (PRISMA) guidelines [31], and the method for the meta-analysis of observational studies in epidemiology [32]. Its protocol was registered in PROSPERO on 10 February 2017, under number CRD42017056521 (http://www.crd.york.ac.uk/PROSPERO).

\section{Research strategy}

Initially, this research focused on both the associations between mental disorders and chronic physical diseases and the association between mental disorders and neurotropic parasites. This last type of association will be presented in a second article. A principal investigator (LOD) performed the article search on Medline, Embase, Lilacs and IENT database (the database of the Institut d'Epidémiologie et de Neurologie Tropicale of the Universite de Limoges [University of Limoges] http://www-ient.unilim. fr/), and manually, from 11 February to 07 May 2017, with no restriction on language or date. The research equation was constructed on PubMed by country as follows: ("Depressive Disorder"[Mesh] OR "Depression" [Mesh] OR "Anxiety Disorders"[Mesh] OR "Anxiety" [Mesh] OR "Bipolar Disorder"[Mesh] OR "Schizophrenia"[Mesh]) AND ("Diabetes metillus"[Mesh] OR "Obesity"[Mesh] OR "Neoplasms"[Mesh] OR "Cardiovascular Diseases"[Mesh] OR "Pulmonary Disease, Chronic Obstructive"[Mesh] OR "Malaria"[Mesh] OR "Cysticercosis"[Mesh] OR "Toxoplasmosis"[Mesh] OR "Toxocariasis"[Mesh] OR "Trypanosomiasis"[Mesh] OR "Chagas Disease"[Mesh]) AND ("Name of a country"[Mesh]).

In Lilacs and Embase, the same keywords were used in free texts to reconstruct the search equation. And in total, 139 different equations corresponding to 139 countries classified as developing and emerging countries according to the World Bank [24] were set-up on each database (Medline, Embase and Lilacs). In the IENT database, articles were searched for free text by entering the term "comorbidity" or "comorbidity in mental health". This 
procedure was adopted because the IENT database is specific to developing and emerging countries. The management (registration and selection) of articles was carried out using Zotéro bibliographic management software.

\section{Inclusion criteria and selection}

To be included in the meta-analysis, each article, which was selected on the basis of its title and then of its summary, had to: be an original article whose full text was available; be a cross-sectional or analytical study; have been conducted on adult patients, both males and females, and on all age groups (age $\geq 15$ years); be a study involving either only hospitalised subjects or only non-hospitalised subjects, but not both hospitalised and non-hospitalised subjects at the same time; specify the method of disease diagnosis. For cross-sectional studies, it had to give the prevalence, or the data from which it could be calculated, and for analytical studies, it had to give the association measures or the data from which they could be calculated.

In case of articles that met the inclusion criteria but were published several times by the same authors, with the same main results, only the most recently published article was included. By "non-hospitalized" we mean patients who live in community or in other words who attended to the health centre (clinic or hospital) for care but who did not stay in the health care centre for one or more nights. We chose to name them this way as opposed to "hospitalized", that is, patients who were staying in a health care centre.

\section{Data extraction and assessment of article quality}

For each article in our study, the principal investigator (LOD) recorded the reference, title, country and continent, the study type and study population, the original disease, the associated disease searched for, and its method of diagnosis, the prevalence or measures of association of the disease searched for (or the data to calculate them), the sample size, sex ratio, mean subject age. The assessment of study quality was performed independently by two investigators (LOD and PEB) using the revised assessment grid of Downs and Black. Each article was assigned a score independently [33, 34] by each investigator and the final score was decided by mutual agreement after comparing the ratings. In the event of a disagreement, the expert opinion of a third investigator (PMP) was requested, without this investigator being aware of the judgments of the first two investigators. The final score was then assigned by mutual agreement by the three investigators.

\section{Statistical analyses}

CMA (Comprehensive Meta Analysis) software Version 3.0 [35] was used for the statistical analyses. Firstly, the potential heterogeneity amongst the selected studies was assessed using statistical $[36,37] \mathrm{Q}$ and $I^{2}$ tests $\left(I^{2}=0\right.$, lack of heterogeneity; $I^{2}<0.25$, low heterogeneity; $I^{2}$ between 0.25 and 0.5, moderate heterogeneity; and $I^{2}>$ 0.5 , significant heterogeneity). The pooled estimates were then calculated using the DerSimonian-Laird random-effects method [38] and the results obtained were presented on a Forest Plot [39] with a significance threshold of $5 \%$.

The investigation for publication bias was performed by constructing a Funnel Plot, the Duval and Tweedie trim and fill test [38] and Egger's linear regression [40]. To assess the robustness of the principal results of our meta-analysis, we carried out a sensitivity analysis by removing the study with the greatest weighting and the studies of lower quality in the pooled studies. The following variables were used to perform subgroup analyses: type of original disease (type of disease a person had before the study) and type of associated diseases (type of diseases investigated through the study), type of included subjects (hospitalised or non-hospitalised) and continent where the study had taken place. Finally, a meta-regression was performed with the study country income level and the quality scores of studies as covariates.

\section{Results}

In total, 2604 articles were found in six languages (English, Spanish, Portuguese, French, Chinese and Russian). After the selection process, 40 articles involving 21,747 participants [41-80] were included in the meta-analysis (Fig. 1) of which 31 articles were prevalence studies [42-46, 48, 50-54, 57, 59-61, 63-65, 67-73, 75-80] and 9 articles were case-control studies [41, 47, 49, 55, 56, 58, 62, 66, 74] (Additional file 1: Table S1 and Additional file 2: Table S2). These articles had been published between 1993 and 2016.

The mean age of the subjects in the prevalence studies was $55.6 \pm 11.7$ years with a range of [ $15.0-99.0$ years] and the female/male sex ratio was 1.0. In the analytical case-control studies, the mean age of the subjects for the cases was $46.2 .0 \pm 11.6$ years and $42.3 \pm 10.4$ years for the controls with a range of [16.0-80.0] years for the two groups. The female/male sex ratio was 0.7 for the case-control studies. The subjects enrolled were hospitalised patients in 13 prevalence studies [46, 48, 50, 53, 54, 57, 64, 67, 71, 76, 78-80] and 3 analytical studies [47, 49, 74]. Non-hospitalised subjects had been studied in 18 prevalence studies $[42-45,51,52,59-61,63,65,68-70,72,73,75,77]$ and 6 analytical studies [41, 55, 56, 58, 62, 66].

Three of the 40 studies had been conducted amongst people with mental disorders who had been screened for chronic physical diseases; two of these were prevalence studies [52, 75] and one was an analytical study [74]. 


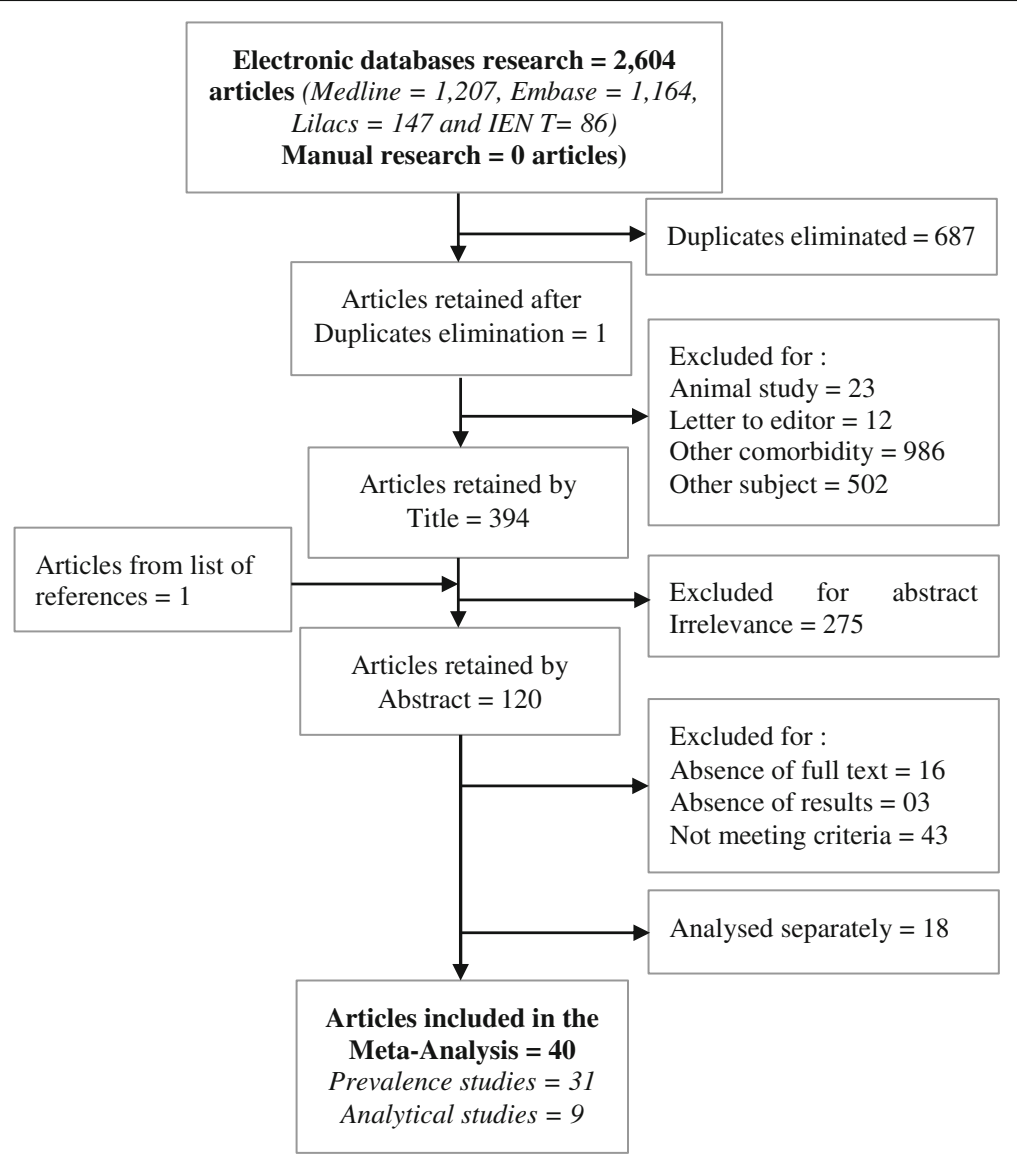

Fig. 1 Flow chart of research strategy of the meta-analysis of comorbidities of mental disorders and chronic physical diseases in developing and emerging countries

Thirty seven studies had been conducted amongst people with chronic physical diseases who were screened for mental disorders; twenty nine of these were prevalence studies $[42-46,48,50,51,53,54,57,59-61,63-$ $65,67-73,76-80]$ and 8 were case-control studies $[41,47,49,55,56,58,62,66]$. There was one multicentre study conducted in Asia amongst the prevalence studies [70] which was considered in our meta-analysis. In total, by continent, we found 1 study in Europe, 4 studies in Africa, 7 studies in America and 46 studies in Asia.

\section{Quality of the studies}

The quality scores of the studies from the Downs and Black assessment grid were a mean of $16.2 \pm 3.2$ out of a maximum of 22 for the prevalence studies and a mean of $15.3 \pm 3.2$ out of a maximum score of 25 for the case-control studies (Additional file 3: Table S3 and Additional file 4: Table S4).

\section{Pooled estimates and heterogeneity}

The diseases found in the prevalence studies were: anxiety and depression for mental disorders; diabetes, obesity, cancer, chronic obstructive pulmonary disease (COPD) and heart disease for the chronic physical diseases. There were 16,462 subjects enrolled in the prevalence studies.

The pooled prevalence of anxiety and/or depression could be estimated from twenty-nine studies involving 16,108 people with chronic diseases (diabetes, obesity, cancer, COPD and heart disease). This pooled prevalence was $36.6 \%$ (95\% CI, 31.4-42.1) as is demonstrated by the forest plot in Fig. 2. There was heterogeneity in the pooled estimates (Q: 795.01, df: $28 ; p<0.0001$ and $I^{2}$ : 96.48). And, the prevalence of diabetes and/or obesity in the 409 people with mental disorders (schizophrenia and/or depression) was assessed as $16.2 \%$ (95\% CI, 7.2-32.5).

The mental disorders found included anxiety, depression, schizophrenia and bipolar disorder. The chronic physical diseases found were chronic coronary heart disease, diabetes, obesity and COPD. With 8 case-control studies (2448 people with chronic diseases and 2422 controls), a pooled odds ratio of 3.1 (95\% CI, 1.8-5.2) (Fig. 3) was found for anxiety and/or depression in people with chronic physical diseases. 


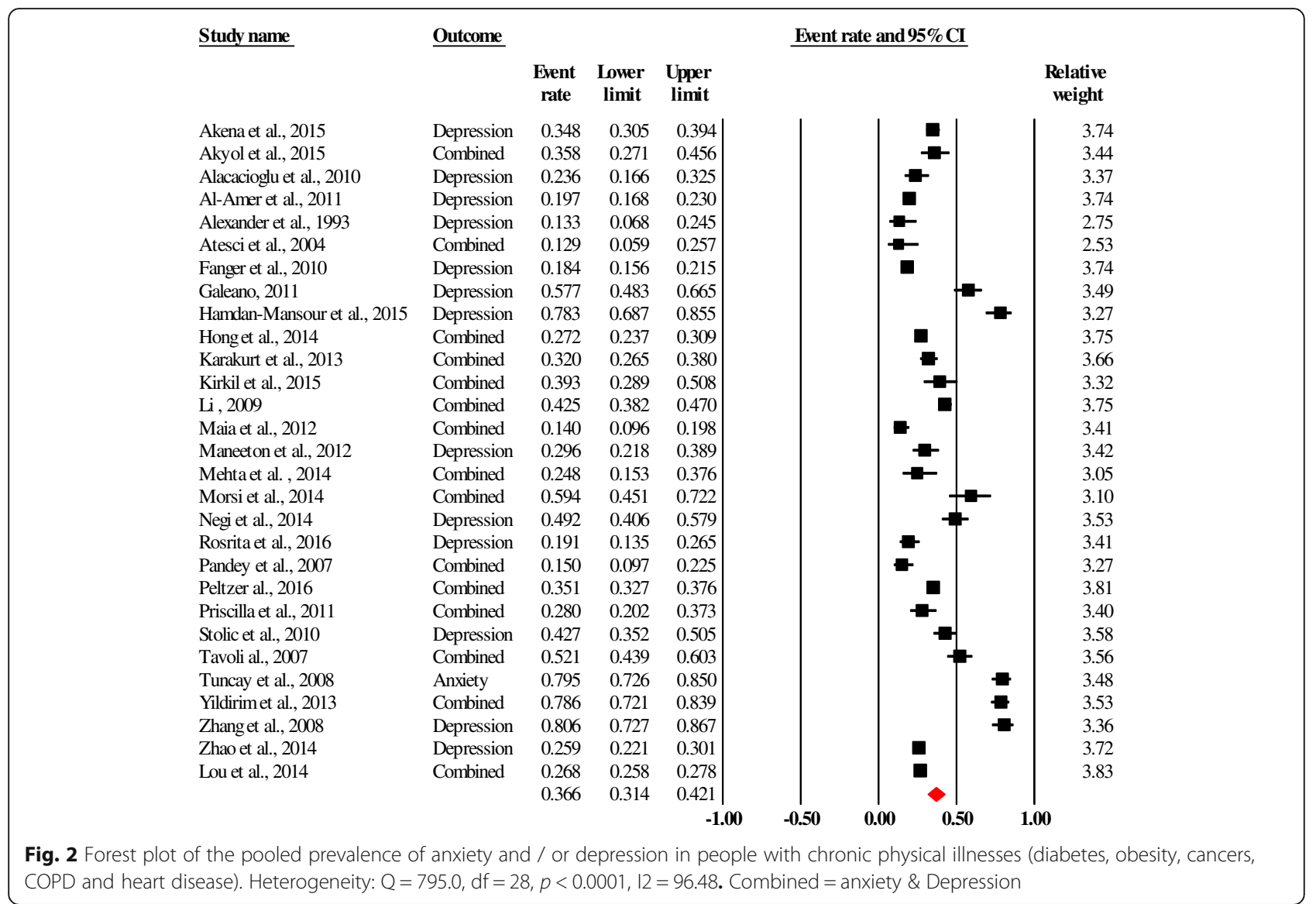

\section{Publication bias}

The distribution of the pooled studies of the various types of comorbidities was illustrated using a Funnel Plot. From the visual Trim and Fill method of Duval and Tweedie, it was estimated that: there were 4 prevalence studies missing for the comorbidities of mental disorders with chronic physical diseases (Fig. 4) and 3 analytical studies were missing for the comorbidities of mental disorders with chronic physical diseases. The existence of this publication bias could be confirmed with the Egger test with the results: Intercept $=2.85(p=0.05)$ and Intercept $=2.75(p=0.14)$, respectively, in the prevalence and analytical studies for the comorbidities of mental disorders with chronic physical diseases.

\section{Sensitivity analysis}

Sensitivity analysis after removing the study with the highest weight on the one hand (Lou et al. study [61]) and low quality study on the other hand (Galeano [51]) from the studies included in the pooled prevalence of

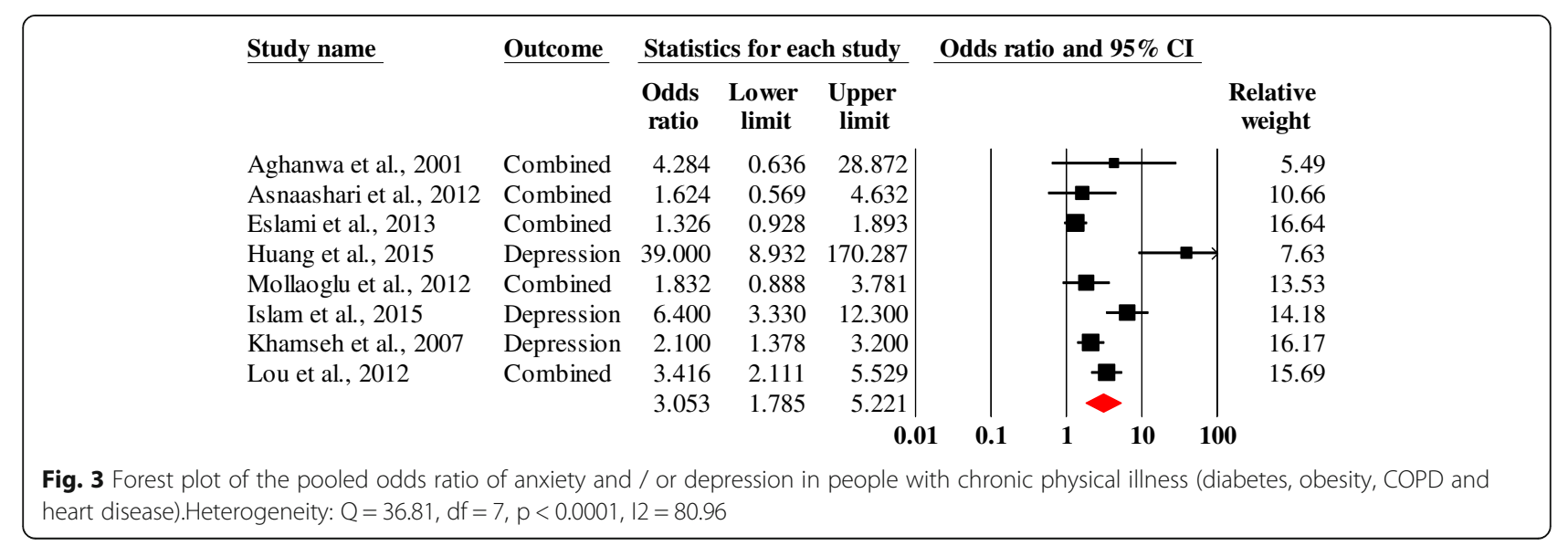




\section{Funnel Plot of Precision by Logit event rate}

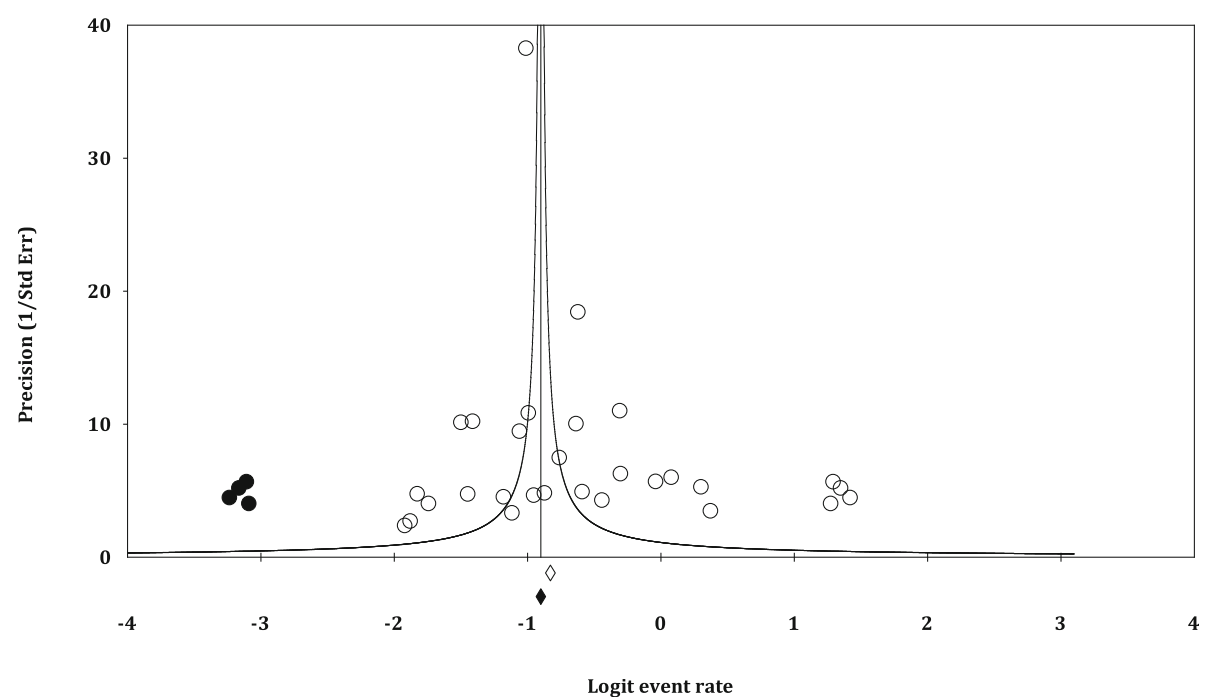

Fig. 4 Funnel plot showing found and missing prevalence studies of comorbidities of mental disorders (anxiety and / or depression) and chronic physical diseases (diabetes, obesity, cancers, COPD and heart disease)

the comorbidities of mental disorders (anxiety and/or depression) with chronic physical diseases (diabetes, obesity, cancer, COPD and heart disease) varied respectively from $36.6 \%$ (95\% CI, 31.4-42.1) to $37.0 \%$ (30.843.6) and $35.9 \%$ (95\% CI, 30.7-41.4). For the analytical studies, the pooled odds ratio for the comorbidities of mental disorders (anxiety and/or depression) with chronic physical diseases (diabetes, obesity, cancer, COPD and heart disease), which was 3.1 (95\% CI, 1.8-5.2), varied to 3.6 (95\% CI, 2.0-6.3) when the study with the highest weight (Eslami et al. [49]) was subtracted and 2.6 (95\% CI, 1.6-4.2) when low quality studies (Asnaashari et al. [47] and Huang et al. [55]) were subtracted. In general, removing the study with the largest weighting on the one hand and low quality on the other hand had little impact on the results.

\section{Subgroup analysis}

Subgroup analysis to determine the pooled estimates by type of disease investigated, type of associated disease, type of subject included (hospitalised or non-hospitalised) and by study continent gave the following results.

The pooled prevalences in people suffering from chronic diseases were: for depression, 36.4\% (95\% CI, 28.9-41.6) and for anxiety and/or depression 33.9\% (95\% CI, 27.1-41.3). A high prevalence of anxiety and/or depression was found: $30.3 \%$ (95\% CI, 22.5-39.6) for cancer, $41.9 \%$ (95\% CI, 28.1-57.0) for diabetes, $40.0 \%$ (95\% CI, 28.7-52.5) for COPD and 50.1\% (95\% CI, 25.874.4) for obesity. If we consider the type of subjects enrolled in the studies, the prevalence of mental disorders in chronic diseases was 39.2\% (95\% CI, 31.1-47.9) in hospitalised subjects and $34.3 \%$ (95\% CI, 27.2-42.2) in non-hospitalised subjects. Most of the studies were concentrated in the Asian continent (24 studies), followed by the American continent ( 3 studies), the African continent ( 2 studies) and the European continent (1 study). The prevalence of comorbidities of mental disorders with chronic diseases was $45.8 \%$ (95\% CI, 25.1-68.1) in Africa, 26.8\% (95\% CI, 14.8-43.5) in America and 37.0\% (95\% CI, 30.9-43.5) in Asia.

Case-control studies on mental disorders with chronic physical diseases were mostly found on the Asian continent with a statistically significant odds ratio of 3.0 (95\% CI, 1.7-5.3). In Africa the association was searched in only one included study [41]. An association of anxiety and/or depression was found in non-hospitalised subjects. By type of disease investigated, the results reveal that the odds ratio of people with chronic physical diseases having depression is 5.8 (95\% CI, 2.4-14.2) and the odds ratio of having both depression and anxiety is 2.1 (95\% CI 1.0-4.2) (Fig. 5). According to the type of original disease, a significant association of 6.4 was found (95\% CI, 1.5-28.0) between obesity and mental disorders (anxiety and depression).

\section{Meta-regression}

The results of the meta-regression about the covariates quality score and type of country obtained by each study are presented below (Table 1). This was only positive for the covariables quality score and income level respectively for the prevalence and analytical studies of comorbidities of mental disorders and chronic physical diseases. 


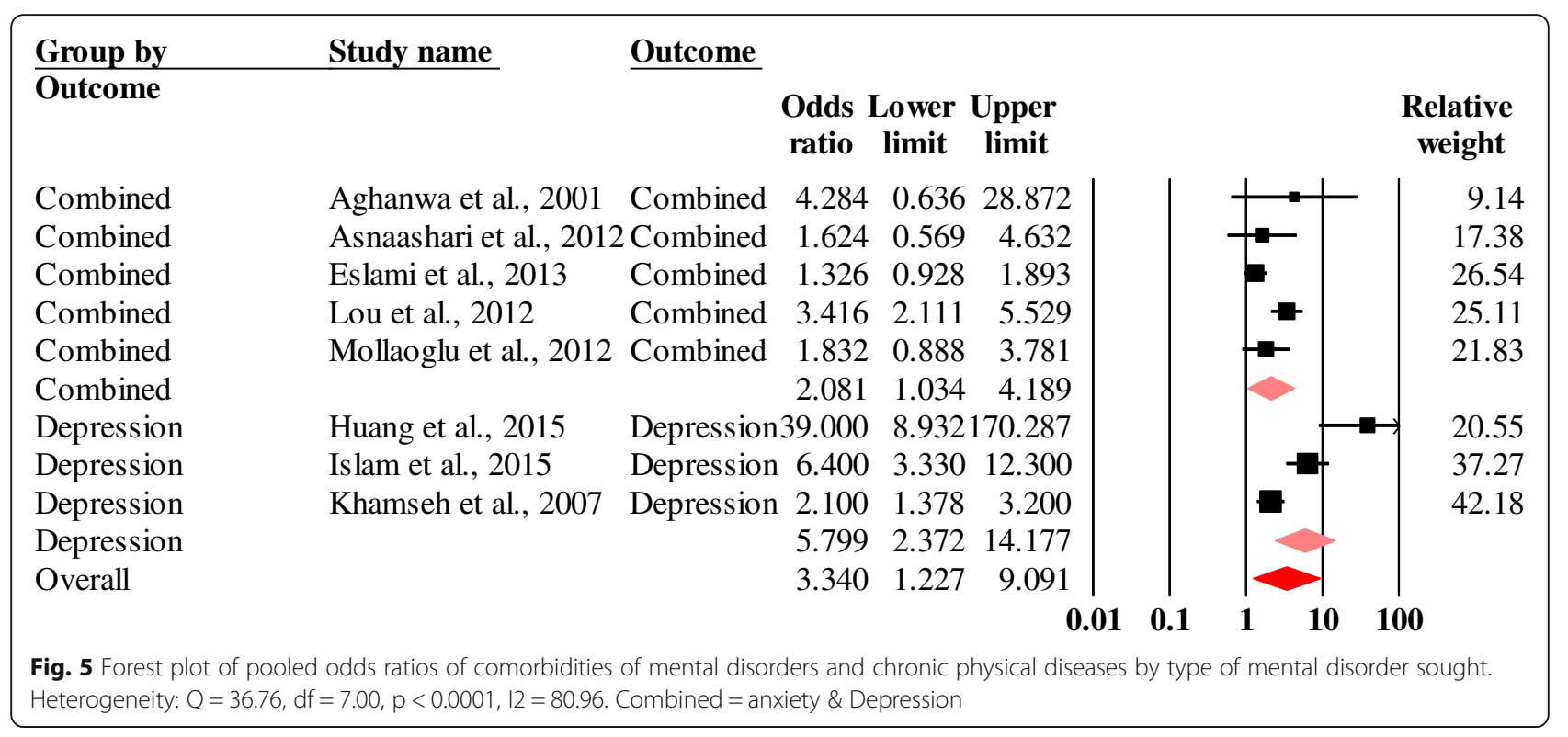

\section{Discussion}

The choice of diseases focused on mental disorders (anxiety, depression, bipolar disorders and schizophrenia) $[5,29]$ and chronic physical diseases (diabetes, obesity, cancer, heart disease and COPD) $[6,30]$ because these represent the greatest cause of deaths worldwide.

In our meta-analysis, the diagnosis of the various diseases in the studies included had been performed by acceptable diagnostic methods (Table 1 and Additional file 1: Table S1 and Additional file 2: Table S2). In fact, the standard diagnostic methods used to diagnose mental disorders had been developed in accordance with DSM-5 (Diagnostic and Statistical Manual for Mental Disorders) and ICD-10 (International Classification of Diseases) [73]. However, as regards the diagnosis of mental disorders, the choice of questionnaire may influence the results. In our studies, Gamze Kirkil et al. demonstrated a 54.5\% prevalence of depression in patients with chronic obstructive pulmonary disease when they performed the diagnosis with the BDI (Beck Depression Inventory) and 63.8\% when the diagnosis had been performed with the HAD-D (Hospital anxiety and depression) [59]. Several other studies point in the same direction [81-84]. Conversely, one study has shown there was no difference in the results between the self-questionnaire and the standardised diagnosis [85]. The diversity of questionnaires found in our studies may be explained by the need to adapt the questionnaire to the population studied $[41,50,54-56,66,68$, $78,80]$ as is recommended. Only $6.5 \%$ of the prevalence studies $[60,63]$ on the comorbidities of anxiety and/or depression in people with chronic physical diseases (cancer, diabetes, obesity, cardiovascular disease and COPD) used a self-assessment questionnaire such as the Self-rating anxiety scale and the Self-rating depression scale. Contrary to the guide to good laboratory practice and quality assurance for laboratory diagnosis which recommends the use of two different diagnostic tests (one very sensitive and the other very specific), the handling of samples by two different technicians unaware of the subject's clinical condition, the studies included in our meta-analysis only considered a single, sensitive, specific test. This is acceptable given the constraints associated with the cost of the analyses. For the chronic diseases, in some studies these were confirmed by following diagnostic guidelines [55] or by choosing people whose disease was already known and confirmed in a specialised care facility.

The assessment of study quality demonstrated that the studies we considered for the meta-analysis were of good

Table 1 Meta-regression according to the Country Income Level and the Study Quality Score

\begin{tabular}{llllllll}
\hline Co-morbidity & Covariate & Number of studies & Coefficient & $Z$ & $p$ & $T^{2}$ \\
\hline 1 & Income Level & 29 & $-0,6286$ & $-0,89$ & 0,3757 & 0,3653 & 0,00 \\
& Quality Score & 29 & 18,492 & 3,00 & 0,0027 & 0,3653 & 0,10 \\
2 & Income Level & 8 & 17,542 & 3,09 & 0,0020 & 0,4177 & 0,23 \\
& Quality Score & 8 & 1100 & 0,89 & 0,3740 & 0,4177 & 0,00 \\
\hline
\end{tabular}

1: Comorbidities of mental disorders and chronic physical diseases of prevalence studies

2: Comorbidity of mental disorders and chronic physical diseases of analytical studies 
quality and that our scores are similar to those obtained by Amerio et al. in their meta-analysis [86]. There was publication bias and significant heterogeneity in our estimates. In fact, the different heterogeneity values obtained indicated that the observed heterogeneities were due to some covariates. Thus, with the help of meta-regression we explained these heterogeneities. However, given the null and insignificant $R^{2}$ values of the meta-regression, it was obvious that there were other covariates which could account for these heterogeneities. For example, the $R^{2}$ of 0.23 found in the comorbidity of mental disorders and chronic physical diseases in the analytical studies indicates that $23 \%$ of the variation found was due to differences in per capita income levels in the 6 countries (China, Iran, Nigeria, Turkey, Bangladesh and Iran) where the 8 included studies were conducted. Despite these attempts at justification, the heterogeneity of the studies included in it remained significant and other covariates could be the reason. In the other types of comorbidities studied, this did not explain the observed variation. Thus, thanks to these results, we can notice that our estimates are not affected by the income level of countries or the quality of studies.

People with mental disorders have a tendency to develop more chronic diseases such as obesity [87] and diabetes [88]. We found a high prevalence, $16.2 \%$, of diabetes and/or obesity in people with schizophrenia or bipolar disorder. Similar results were found in a metaanalysis carried out in Canada with an obesity prevalence of $19 \%$ in people with depression. Our result in a hospital setting is also close to the prevalence of type 2 diabetes in schizophrenic patients found in a retrospective study conducted over 6 years in the United States [89] and the prevalence of obesity found in the general Canadian population of $12 \%$. Conversely, our prevalence is less than those of type 2 diabetes, found in $26 \%$ of people with bipolar disorders in the United States [89] and obesity, found in $42.1 \%$ of patients with schizophrenia in Canada [90]. It is higher than the $6.7 \%$ of diabetics found in a study in Belgium in patients with bipolar disorders [91]. These studies show that the prevalence of diabetes metillus is 4 to 5 times higher in people with schizophrenia [92] and that of type 2 diabetes is 3 times higher in the subpopulation of people with bipolar disorders [88] compared to the prevalence in the general population. Despite the reduced number of studies, the significance level of our result conveys the alarming nature of chronic physical diseases for people with mental disorders. It also shows how important and necessary their diagnosis and early management is, especially in developing and emerging countries.

In contrast to the insufficient number of studies found for chronic diseases in people with mental disorders in our meta-analysis, some meta-analyses involving multiple countries in North America, Europe, and Asia [84, 93-96] do have data showing that people with mental disorders have an increased risk of developing chronic physical diseases. This lack of data in developing and emerging countries may be explained by the lack of medical consultations for people with mental disorders and also by the rather reduced number of health centres which, when they exist, are not accessible [97]. In addition, the influence of religion also has a significant impact on the diagnosis of mental disorders in developing and emerging countries. Often wrongly attributed to a spiritual effect, $80 \%$ of people with mental disorders and their families prefer to consult religious leaders or healers or exorcistpriests [98]. The lack of financial means also aggravates this situation and is an obstacle to consultation for people who wish to access the appropriate services [99].

Several studies in this meta-analysis considered mental disorders in people with chronic diseases such as cancer, diabetes, obesity, cardiovascular disease and COPD. This is because the policy in developing and emerging countries in this epidemiological transition period favours fighting non-communicable diseases [3]. The prevalence of depression in people with chronic diseases is in line with the results of the meta-analysis by Solano et al. [100] which shows a prevalence of depression of between 13 and $79 \%$ in people with chronic physical diseases (cancer, heart disease, renal disease and COPD). The difference in the prevalences for mental disorders comorbidities in people with chronic diseases depends on the "hospitalised" or "non-hospitalised" status of the subject and may be due to their health status. This is often more severe in hospitalised patients for whom there is a higher prevalence (which seems logical). Similarly, Ren et al. [101] in their meta-analysis on depression and coronary heart disease found pooled prevalences of $51 \%$ (95\% CI, 43-58) for depression in coronary disease in hospitalised patients and prevalences of 34.6 to $45.8 \%$ in non-hospitalised patients.

In addition, our meta-analysis revealed that the increased risk for anxiety and/or depression in people with chronic physical diseases was 310\% (95\% CI, 1.8-5.2). The results of meta-analyses in other countries with lower estimates give odds ratios of: 1.27 to 1.55 for depression associated with obesity in hospital [102-104] and an odds ratio of $1.4(95 \% \mathrm{CI}, 1.2-1.6)$ for anxiety associated with obesity [105].

Despite efforts made to minimize bias in our metaanalysis, it still had some limitations, mostly linked to the included studies. These limitations mainly involve: the size of the various samples, which were often relatively small; the diagnosis of mental disorders not being confirmed by a specialist in some studies; and the significant number of studies performed in Asia, which could be an obstacle to generalising the results to all 
developing and emerging countries. In addition, some estimates could not be determined because of a lack of studies which met our inclusion criteria, especially for chronic diseases comorbidities in people with mental disorders. Mental disorders are also very common during the perinatal period. This is a criterion that could have been included in our inclusion criteria. But the objective of this meta-analysis was oriented towards general results. So, this type of specificity has not been considered. Finally, the fact that we chose to perform our sensitivity test by removing the studies with the greatest weighting could be considered a limitation by those who prefer to subtract the poorer quality studies when performing their sensitivity test. However, it is generally recommended that the weighting of the studies be considered as we have done here.

Nonetheless, our meta-analysis is the first to involve only developing and emerging countries for the comorbidities of mental disorders and chronic physical diseases It was performed following the PRISMA 2015 guidelines and the protocol was registered in the PROSPERO international prospective register of systematic reviews database. Finally, with the performance of the meta-regression, it was possible to show that the quality of studies included in our meta-analysis was above average and did not affect our pooled estimates.

\section{Conclusion}

In conclusion, our pooled estimates show that the comorbidities of mental disorders and chronic physical diseases are indeed a real burden for people in developing and emerging countries. Given the difficulties in developing and emerging countries, prioritisation of the mental health sector is essential in these countries experiencing the increasing burden of non-communicable diseases because there is "No health without mental health" [106]. In addition, awareness of mental health must be integrated into all aspects of health and social policy, into the planning of the healthcare system and into the provision of primary \& secondary healthcare. Finally, policies and healthcare for mental health must be reoriented to place more emphasis on the simultaneous diagnosis and treatment of mental disorders and other associated diseases. Through this first meta-analysis in developing and emerging countries, we hope to open the way for other studies, especially cohort studies on comorbidities in mental health. More specifically, studies on subjects with mental disorders in whom chronic physical diseases will be investigated because of the lack of specific data on this comorbidity. In Oceanic, African, and American countries, it is essential to develop research on these co-morbidities.

\section{Additional files}

Additional file 1: Table S1. Characteristics of Prevalence Studies of Comorbidities of Mental Disorders with Chronic Physical Diseases. MINI: Mini International Neuropsychiatric Interview, HADS: Hospital Anxiety and Depression Scale, BDI: Beck Depression Inventory, STAI: State-Trait Anxiety Inventory, PHQ: Patient Health Questionnaire, DSM: Diagnostic and Statistical Manual for Mental Disorders, SCIDI/CV: Structured Clinical Interview for DSM-IV_Clinical Version; PSE: Present State Examination, MMSE: Mini Mental State Examination, SADS-L: Schedule for Affective Disorders and Schizophrenia-Lifetime, MSE: Mental Status Examination, SAS: Selfrating anxiety scale, SDS: Self-rating depression scale, HAMA: Hamilton Anxiety Rating Scale, HAMD: Hamilton Depression Rating Scale, ICD: International Classification of Diseases, HAD-D: Hospital anxiety and depression, FBG: Fasting Blood Glucose; HbA1c: Glycated Hemoglobin, COPD: Chronic Obstructive Pulmonary Disease, CVD: CerebroVascular Disease, F: Female, M: male, Age in years. (DOCX 149 kb)

Additional file 2: Table S2. Characteristics of Analytical Studies of Comorbidities of Mental Disorders with Chronic Physical Diseases. COPD: Chronic Obstructive Pulmonary Disease, GHQ: General Health Questionnaire, PSE: Present State Examination, ICD: International Classification of Diseases, IDF: International Diabetes Federation, SCL: Syndrome Checklist, SCL: Syndrome Checklist, HADS: Hospital Anxiety and Depression Scale, BDI: Beck Depression Inventory, PHQ: Patient Health Questionnaire, BAl: Beck Anxiety Inventory, BAE: Beck Depression Inventory, F: Female, M: male, Age in years. (DOCX $129 \mathrm{~kb}$ )

Additional file 3: Table S3. Characteristics of quality scores of prevalence studies. Global quality (Items: 1-2-3-5-6-7-9-10); External validity (Items:1112-13); Results bias (Items: 15-16-18-20); Confusion and selection bias (Item: 25); Power (Item: 27) and S: Quality score. (DOCX 71 kb)

Additional file 4: Table S4. Characteristics of quality scores of analytical studies. Global quality (Items: 1-2-3-5-6-7-9-10); External validity (Items:1112-13); Results bias (Items: 15-16-18-20); Confusion and selection bias (Item: 25); Power (Item: 27) and S: Quality score. (DOCX 30 kb)

\section{Acknowledgements}

Not applicable.

\section{Funding}

LOD was an intern at Sanofi for six months during which he received a grant. Sanofi also provided access to the electronic databases and assistance with the finalization of the article.

\section{Availability of data and materials}

All data generated or analyzed in this article is presented in the additional information file and available from the Correspondence Author (LOD).

\section{Authors' contributions}

$L O D$, contributed to the drafting of the research protocol, its registration in PROSPERO, the analysis of the method, the research - selection - evaluation of the quality of the articles, the analysis of the data, the interpretation of the data and the preparation of the manuscript. PEB contributed to the finalization of the research protocol, the evaluation of the quality of the articles and the preparation of the manuscript. DG contributed to the finalization of the research protocol and the preparation of the manuscript. $\mathrm{VL}$ contributed to the finalization of the research protocol and the preparation of the manuscript. BM and FB contributed to the finalization of the research protocol, the analysis of the method and the preparation of the manuscript. PMP contributed to the looking for funding, the design of the study, the writing of the research protocol, the analysis of the method, the evaluation of the quality of the articles, the analysis of the data, the interpretation of the data and the preparation of the manuscript. This manuscript has been read and approved by all authors.

\section{Ethics approval and consent to participate}

Not applicable.

Consent for publication

Not applicable. 


\section{Competing interests}

$V L$ and PEB are Sanofi employees and own Sanofi stocks. DG is a former employee of Sanofi. All other authors declare that there is no conflict of interest related to the writing and publication of this article.

\section{Publisher's Note}

Springer Nature remains neutral with regard to jurisdictional claims in published maps and institutional affiliations.

\section{Author details}

'INSERM, Univ. Limoges, CHU Limoges, UMR_S 1094, Tropical

Neuroepidemiology, Institute of Neuroepidemiology and Tropical Neurology, CNRS FR 3503 GEIST, F-87000 Limoges, France. ${ }^{2}$ Access to Medicines, SANOFI, SAG / CSVB, 82 AV Raspail, 94250 Gentilly, France.

\section{Received: 6 April 2018 Accepted: 5 March 2019}

Published online: 13 March 2019

\section{References}

1. Thornicroft G, Votruba N. Millennium development goals: lessons for global mental health. Epidemiol Psychiatr Sci. 2015;24:458-60.

2. Thornicroft G, Votruba N, Baingana F, Bhugra D, Eaton J, Goetzke K, Gureje O, Ivbijaro G, Lamichhane J, Lund C, et al. Millennium development goals: lessons for global mental health. Epidemiol Psychiatr Sci. 2014;24:458-60.

3. Vos T, Allen C, Arora M, Barber RM, Bhutta ZA, Brown A, Carter A, Casey DC, Charlson FJ, Chen AZ, et al. Global, regional, and national incidence, prevalence, and years lived with disability for 310 diseases and injuries, 1990-2015: a systematic analysis for the global burden of disease study 2015. Lancet. 2016;388:1545-602.

4. Whiteford HA, Degenhardt L, Rehm J, Baxter AJ, Ferrari AJ, Erskine HE, Charlson FJ, Norman RE, Flaxman AD, Johns N, et al. Global burden of disease attributable to mental and substance use disorders: findings from the Global Burden of Disease Study 2010. Lancet (London, England). 2013; 382:1575-86.

5. Vigo D, Thornicroft G, Atun R. Estimating the true global burden of mental illness. Lancet Psychiatry. 2016:3:171-8.

6. Yach D, Hawkes C, Gould CL, Hofman KJ. The global burden of chronic diseases: overcoming impediments to prevention and control. JAMA. 2004; 291:2616-22.

7. World Health Organization. World Health Report 2001 - Mental Health: New Design, New Hope. WHO. Geneva: WHO library; 172p. 2001. https://www. who.int/whr/2001/media_centre/press_release/en/. Accessed 13 Sept 2017.

8. World Health Organization. Chapitre 2: Impact of mental and behavioural disorders. http://www.who.int/whr/2001/chapter2/fr/index5.html. Accessed 24 Apr 2017

9. Haute Autorité de Santé: Guidance Note. Multi-annual programme on psychiatry and mental health. 2013. http://www.has-sante.fr/portail/upload/ docs/application/pdf/2014-01/2013_10_08_programme_sante_mentale_ college.pdf. Accessed 24 Apr 2017.

10. World Health Organization: Chronic diseases. http://www.who.int/topics/ chronic diseases/fr/. Accessed 4 May 2017.

11. World Health Organization: Facing the Facts \#1: Chronic Diseases and Their Common Risk Factors. http://www.who.int/chp/chronic_disease_report/ media/Factsheet1.pdf. Accessed 23 Apr 2017.

12. World Health Organization: 10 facts on non-communicable diseases. 10 faits sur les maladies non transmissibles. Accessed 23 Apr 2017.

13. Centers for Disease Control and prevention - National Center for Chronic Diseases Prevention and Health Promotion: Mental Health and Chronic Diseases. National Healthy Worksites (NHW). https://www.cdc.gov/ workplacehealthpromotion/tools-resources/pdfs/issue-brief-no-2-mentalhealth-and-chronic-disease.pdf (2012). Accessed 18 Sep 2016.

14. Nakash O, Levav I, Aguilar-Gaxiola S, Alonso J, Andrade LH, Angermeyer MC, Bruffaerts R, Caldas-de-Almeida JM, Florescu S, de Girolamo G, et al. Comorbidity of common mental disorders with cancer and their treatment gap: findings from the world mental health surveys. Psycho-Oncology. 2014; 23:40-51.

15. World Health Organization. Invest in mental health. Department of Mental Health and Substance Abuse Noncommunicable Diseases and Mental Health, Geneva: WHO library; 2004 49p. http://www.who.int/iris/handle/ 10665/42896. Accessed 6 Nov 2016.
16. Alves FMB, Coutinho MSSA, Sakae TM, Cosentino MB. Cardiovascular risk factors in patients with non-coronarian atherosclerotic disease in hospital in the South of Brazil: case-control study. Rev Soc Bras Clin Med. 2009;7:3-10.

17. Al-Hayek AA, Robert AA, Alzaid AA, Nusair HM, Zbaidi NS, Al-Eithan MH, Sam AE. Association between diabetes self-care, medication adherence, anxiety, depression, and glycemic control in type 2 diabetes. Saudi Med J. 2012;33:681-3.

18. Danna SM, Graham E, Burns RJ, Deschênes SS, Schmitz N. Association between depressive symptoms and cognitive function in persons with diabetes mellitus: a systematic review. PLoS One. 2016;11:e0160809.

19. Lunel C. M.D: Diabète et maladies mentales. In Fédération des familles et amis de la personne atteinte de maladie mentale.2012. http://www. ffapamm.com/nouvelle/article-diabete-et-maladies-mentales 7355. Accessed 18 Sep 2016.

20. Mejía-Arango S, Zúñiga-Gil C. Diabetes mellitus as a risk factor for dementia in the Mexican elder population. Rev Neurol. 2011;53:397-405.

21. Esposito M, Gallai B, Roccella M, Marotta R, Lavano F, Lavano SM, Mazzotta G, Bove D, Sorrentino M, Precenzano F, et al. Anxiety and depression levels in prepubertal obese children: a case-control study. Neuropsychiatr Dis Treat. 2014;10:1897-902.

22. Yohannes AM, Müllerová H, Hanania NA, Lavoie K, Tal-Singer R, Vestbo J, Rennard SI, Wouters EFM. Long-term course of depression trajectories in patients with COPD: a 3-year follow-up analysis of the evaluation of COPD longitudinally to identify predictive surrogate endpoints cohort. Chest. 2016; 149:916-26.

23. Zhang MWB, Ho RCM, Cheung MWL, Fu E, Mak A. Prevalence of depressive symptoms in patients with chronic obstructive pulmonary disease: a systematic review, meta-analysis and meta-regression. Gen Hosp Psychiatry. 2011:33:217-23.

24. World Bank : Country and Lending Groups. http://data.worldbank.org/ income-level/low-and-middle-income (2016). Accessed 18 Sep 2016.

25. Aydin IO, Uluşahin A. Depression, anxiety comorbidity, and disability in tuberculosis and chronic obstructive pulmonary disease patients: applicability of GHQ-12. Gen Hosp Psychiatry. 2001;23:77-83.

26. Asghar S, Hussain A, Ali SMK, Khan AKA, Magnusson A. Prevalence of depression and diabetes: a population-based study from rural Bangladesh. Diabet Med. 2007:24:872-7.

27. Doherty AM, Gaughran F. The interface of physical and mental health. Soc Psychiatry Psychiatr Epidemiol. 2014;49:673-82.

28. Mbewe EK, Uys LR, Birbeck GL. The impact of a short depression and anxiety screening tool in epilepsy Care in Primary Health Care Settings in Zambia. Am J Trop Med Hyg. 2013;89:873-4.

29. Aillon J-L, Ndetei DM, Khasakhala L, Ngari WN, Achola HO, Akinyi S, Ribero S. Prevalence, types and comorbidity of mental disorders in a Kenyan primary health Centre. Soc Psychiatry Psychiatr Epidemiol. 2014;49:1257-68.

30. Blakemore A, Dickens C, Guthrie E, Bower P, Kontopantelis E, Afzal C, Coventry PA. Depression and anxiety predict health-related quality of life in chronic obstructive pulmonary disease: systematic review and meta-analysis. Int J Chron Obstruct Pulmon Dis. 2014;9:501-12.

31. Moher D., Shamseer L., Ghersi D., Liberati A., Petticrew M., Shekelle P., et al. PRISMA for systematic review protocols (PRISMA-P). http://prisma-statement. org/Extensions/Protocols.aspx. Accessed 30 Oct 2016.

32. Stroup DF, Berlin JA, Morton SC, Olkin I, Williamson GD, Rennie D, Moher D, Becker BJ, Sipe TA, Thacker SB. Meta-analysis of observational studies in epidemiology: a proposal for reporting. Meta-analysis of observational studies in epidemiology (MOOSE) group. JAMA. 2000;283:2008-12.

33. National Collaborating Centre for Methods and Tools. Quality Checklist for Health Care Intervention Studies. Hamilton, On: McMaster University : Metaanalysis in medical research. (2008). http://www.nccmt.ca/resources/search/9. Accessed 23 Mar 2017.

34. Downs SH, Black N. The feasibility of creating a checklist for the assessment of the methodological quality both of randomised and non-randomised studies of health care interventions. J Epidemiol Community Health. 1998:52:377-84.

35. CMA Manuals: Comprehensive Meta-Analysis Software. (2017). https://www. meta-analysis.com/pages/cma_manual.php. Accessed 31 Mar 2017.

36. Borenstein M, Larry VH, Julian PTH, Hannah RR. Introduction to MetaAnalysis. Oxford: Wiley; 2009.

37. Boyle MH. Guidelines for evaluating prevalence studies. Evid Based Ment Health. 1998:1:37-9.

38. Borenstein M, Hedges LV, Higgins JPT, Rothstein HR. A basic introduction to fixed-effect and random-effects models for meta-analysis. Res Synth Methods. 2010;1:97-111. 
39. Borenstein M, Larry VH, Julian PTH, Hannah RR. Introduction to forest plots. Oxford: Wiley; 2009.

40. Egger M, Davey Smith G, Schneider M, Minder C. Bias in meta-analysis detected by a simple, graphical test. BMJ. 1997;315:629-34.

41. Aghanwa HS, Erhabor GE. Specific psychiatric morbidity among patients with chronic obstructive pulmonary disease in a Nigerian general hospital. J Psychosom Res. 2001;50:179-83.

42. Akena D, Kadama P, Ashaba S, Akello C, Kwesiga B, Rejani L, Okello J, Mwesiga EK, Obuku EA. The association between depression, quality of life, and the health care expenditure of patients with diabetes mellitus in Uganda. J Affect Disord. 2015;174:7-12.

43. Akyol M, Ulger E, Alacacioglu A, Kucukzeybek Y, Yildiz Y, Bayoglu V, Gumus Z, Yildiz I, Salman T, Varol U, et al. Sexual satisfaction, anxiety, depression and quality of life among Turkish colorectal cancer patients [lzmir oncology group (IZOG) study]. Jpn J Clin Oncol. 2015;45:657-64.

44. Alacacioglu A, Binicier O, Gungor O, Oztop I, Dirioz M, Yilmaz U. Quality of life, anxiety, and depression in Turkish colorectal cancer patients. Support Care Cancer. 2010;18:417-21.

45. Al-Amer RM, Sobeh MM, Zayed AA, Al-Domi HA. Depression among adults with diabetes in Jordan: risk factors and relationship to blood sugar control. J Diabetes Complicat. 2011;25:247-52

46. Alexander PJ, Dinesh N, Vidyasagar MS. Psychiatric morbidity among cancer patients and its relationship with awareness of illness and expectations about treatment outcome. Acta Oncologica (Stockholm, Sweden). 1993:32:623-6

47. Asnaashari AMH, Talaei A, Haghigh B. Evaluation of psychological status in patients with asthma and COPD. Iran J Allergy Asthma Immunol. 2012;11:65-71.

48. Atesci FC, Baltalarli B, Oguzhanoglu NK, Karadag F, Ozdel O, Karagoz N. Psychiatric morbidity among cancer patients and awareness of illness. Support Care Cancer. 2004;12:161-7.

49. Eslami B, Sundin O, Macassa G, Khankeh HR, Soares JJF. Anxiety, depressive and somatic symptoms in adults with congenital heart disease. J Psychosom Res. 2013;74:49-56.

50. Fanger PC, Azevedo RCS, Mauro MLF, Lima DD, Gaspar KC, Silva VF, Nascimento WTJ, Botega NJ. Depression and suicidal behavior of cancer inpatients: prevalence and associated factors. Revista Da Associacao Medica Brasileira (1992). 2010;56:173-8.

51. Galeano MT. Depressive indicators in a sample of patients with obesity. Eureka (Asunción, En LíneaEureka). 2011;8:254-66.

52. Gomes FA, Almeida KM, Magalhaes PV, Caetano SC, Kauer-Sant'Anna M, Lafer B. F: cardiovascular risk factors in outpatients with bipolar disorder: a report from the Brazilian research network in bipolar disorder^ien. Rev Bras Psiquiatr. 2013;35:126-30.

53. Hamdan-Mansour AM, Al Abeiat DD, Alzoghaibi IN, Ghannam BM, Hanouneh SI. Psychosocial and sociodemographic correlates of life satisfaction among patients diagnosed with cancer in Jordan. J Cancer Educ. 2015;30:31-6.

54. Hong JS, Tian J. Prevalence of anxiety and depression and their risk factors in Chinese cancer patients. Support Care Cancer. 2014;22:453-9.

55. Huang Y, Chen J, Yang J, Song K, Wang X, Cheng X, Qu S. Evaluation of depressive symptoms in obese patients with or without acanthosis nigricans. Hormones (Athens, Greece). 2015;14:417-24.

56. Islam SMS, Ferrari U, Seissler J, Niessen L, Lechner A. Association between depression and diabetes amongst adults in Bangladesh: a hospital based case-control study. J Glob Health. 2015;5:020406.

57. Karakurt $P$, Ünsal A. Fatigue, anxiety and depression levels, activities of daily living of patients with chronic obstructive pulmonary disease. Int J Nurs Pract. 2013;19:221-31.

58. Khamseh ME, Baradaran HR, Rajabali H. Depression and diabetes in Iranian patients: a comparative study. Int J Psychiatry Med. 2007;37:81-6.

59. Kirkil G, Deveci F, Deveci SE, Atmaca M. Anxiety and depression symptoms in patients with chronic obstructive pulmonary disease (copd). Klinik Psikofarmakoloji Bulteni. 2015;25:151-61.

60. Li A. Analyses on the rate and epidemic characteristics of anxiety and depression among cancer patients in Yangpu District in Shanghai. Asian Pac J Cancer Prev. 2009;10:895-8.

61. Lou P, Chen P, Zhang P, Yu J, Wang Y, Chen N, Zhang L, Wu H, Zhao J. Effects of smoking, depression, and anxiety on mortality in COPD patients: a prospective study. Respir Care. 2014;59:54-61.

62. Lou P, Zhu Y, Chen P, Zhang P, Yu J, Zhang N, Chen N, Zhang L, Wu H, Zhao J. Prevalence and correlations with depression, anxiety, and other features in outpatients with chronic obstructive pulmonary disease in China: a cross-sectional case control study. BMC Pulm Med. 2012;12:53.

63. Maia ACCO, Braga AA, Brouwers A, Nardi AE, Oliveira e Silva AC. Prevalence of psychiatric disorders in patients with diabetes types 1 and 2. Compr Psychiatry. 2012;53:1169-73.

64. Maneeton B, Maneeton N, Mahathep P. Prevalence of depression and its correlations: a cross-sectional study in Thai cancer patients. Asian Pac J Cancer Prev. 2012;13:2039-43.

65. Mehta JR, Ratnani IJ, Dave JD, Panchal BN, Patel AK, Vala AU. Association of psychiatric co-morbidities and quality of life with severity of chronic obstructive pulmonary disease. East Asian Arch Psychiatr. 2014;24:148-55.

66. Mollaoglu H, Ucok K, Kaplan A, Genc A, Mayda H, Guzel HI, Sener U, Uygur E, Ozbulut O. Association analyses of depression, anxiety, and physical fitness parameters in Turkish obese adults. J Back Musculoskelet Rehabil. 2012;25:253-60.

67. Morsi TS, Ghobashy S, Younis G. Quality of life and psychological disorders in Egyptian patients with chronic lung diseases: Clinico-physiological correlation. Egypt J Chest Dis Tuberc. 2014;63:731-43.

68. Negi H, Sarkar M, Raval AD, Pandey K, Das P. Presence of depression \& its risk factors in patients with chronic obstructive pulmonary disease. Indian J Med Res. 2014;139:402-8.

69. Pandey M, Devi N, Thomas BC, Kumar SV, Krishnan R, Ramdas K. Distress overlaps with anxiety and depression in patients with head and neck cancer. Psycho-Oncology. 2007;16:582-6.

70. Peltzer K, Pengpid S. Anxiety and depressive features in chronic disease patients in Cambodia, Myanmar and Vietnam. S Afr J Psychiatry. 2016;22:1-4.

71. Priscilla D, Hamidin A, Azhar MZ, Noorjan KON, Salmiah MS, Bahariah K. Assessment of depression and anxiety in haematological cancer patients and their relationship with quality of life. East Asian Arch Psych. 2011;21:108-14.

72. Rosrita NN, Yunus F, Ginting T, Nurwidya F. Depression in patients with stable chronic obstructive pulmonary disease: a cross-sectional study in the national center for respiratory diseases in Indonesia. Pneumologia (Bucharest, Romania). 2016;61:92-6.

73. Stolic RV, Trajkovic GZ, Mihailovic B, Sipic MV, Celic DB, Lazic SF, Nikolic GR, Sovtic SR, Stolic DZ. Characteristics of depression in obese people living in an insecure environment. Indian J Med Sci. 2010;64:307-14.

74. Subashini R, Deepa M, Padmavati R, Thara R, Mohan V. Prevalence of diabetes, obesity, and metabolic syndrome in subjects with and without schizophrenia (CURES-104). J Postgrad Med. 2011;57:272-7.

75. Sweileh WM, Dalal SA, Zyoud SH, Al-Jabi SW, Al-Ali I. Diabetes mellitus in patients with schizophrenia in West-Bank, Palestine. Diabetes Res Clin Pract. 2013;99:351-7.

76. Tavoli A, Mohagheghi MA, Montazeri A, Roshan R, Tavoli Z, Omidvari S. Anxiety and depression in patients with gastrointestinal cancer: does knowledge of cancer diagnosis matter? BMC Gastroenterol. 2007;7:28.

77. Tuncay T, Musabak I, Gok DE, Kutlu M. The relationship between anxiety, coping strategies and characteristics of patients with diabetes. Health Qual Life Outcomes. 2008;6:79.

78. Yildirim A, Hacihasanoğlu Aşilar R, Bakar N, Demir N. Effect of anxiety and depression on self-care agency and quality of life in hospitalized patients with chronic obstructive pulmonary disease: a questionnaire survey. Int J Nurs Pract. 2013;19:14-22.

79. Zhang J, Ye M, Huang H, Li L, Yang A. Depression of chronic medical inpatients in China. Arch Psychiatr Nurs. 2008;22:39-49.

80. Zhao L, Li X, Zhang Z, Song C, Guo C, Zhang Y, Zhang Y, Li L, Lu G, Zheng $\mathrm{G}$, et al. Prevalence, correlates and recognition of depression in Chinese inpatients with cancer. Gen Hosp Psychiatry. 2014;36:477-82.

81. Anderson RJ, Freedland KE, Clouse RE, Lustman PJ. The prevalence of comorbid depression in adults with diabetes: a meta-analysis. Diabetes Care. 2001;24:1069-78.

82. Barnard KD, Skinner TC, Peveler R. The prevalence of co-morbid depression in adults with type 1 diabetes: systematic literature review. Diab Med. 2006; 23:445-8.

83. Krebber $A M H$, Buffart $L M$, Kleijn $G$, Riepma IC, de Bree $R$, Leemans $C R$, Becker A, Brug J, van Straten A, Cuijpers P, et al. Prevalence of depression in cancer patients: a meta-analysis of diagnostic interviews and self-report instruments. Psycho-Oncology. 2014;23:121-30.

84. Stubbs B, Vancampfort D, De Hert M, Mitchell AJ. The prevalence and predictors of type two diabetes mellitus in people with schizophrenia: a systematic review and comparative meta-analysis. Acta Psychiatr Scand. 2015;132:144-57. 
85. Yang Y-L, Liu L, Wang Y, Wu H, Yang X-S, Wang J-N, Wang L. The prevalence of depression and anxiety among Chinese adults with cancer: a systematic review and meta-analysis. BMC Cancer. 2013;13:393.

86. Amerio A, Stubbs B, Odone A, Tonna M, Marchesi C, Nassir Ghaemi S. Bipolar I and II Disorders; A Systematic Review and Meta-Analysis on Differences in Comorbid Obsessive-Compulsive Disorder. Iran J Psychiatry Behav Sci. 2016;10(3):e3604.

87. Joffre-Velázquez VM, García-Maldonado G, Martínez-Perales G. Sald ívarGonzalez AgH: Revisión sistemática de la asociación entre sobrepeso, obesidad y enfermedad mental, conénfasis en el trastorno esquizofrénico. Rev colomb psiquiatr. 2009;38:705-16.

88. Mcintyre R, Konarski J, Misener V, Kennedy S. Bipolar disorder and diabetes mellitus: epidemiology, etiology, and treatment implications. Ann Clin Psychiatry. 2005;17:83-93.

89. Regenold WT, Thapar RK, Marano C, Gavirneni S, Kondapavuluru PV. Increased prevalence of type 2 diabetes mellitus among psychiatric inpatients with bipolar I affective and schizoaffective disorders independent of psychotropic drug use. J Affect Disord. 2002;70:19-26.

90. Coodin S. Body mass index in persons with schizophrenia. Can J Psychiatry. 2001;46:549-55.

91. Van Winkel R, De Hert M, Van Eyck D, Hanssens L, Wampers M, Scheen A, Peuskens J. Prevalence of diabetes and the metabolic syndrome in a sample of patients with bipolar disorder. Bipolar Disord. 2008;10:342-8.

92. De Hert M, van Winkel R, Van Eyck D, Hanssens L, Wampers M, Scheen A, Peuskens J. Prevalence of diabetes, metabolic syndrome and metabolic abnormalities in schizophrenia over the course of the illness: a crosssectional study. Clin Pract Epidemiol Mental Health. 2006;2:14.

93. Laursen TM, Munk-Olsen T, Agerbo E, Gasse C, Mortensen PB. Somatic hospital contacts, invasive cardiac procedures, and mortality from heart disease in patients with severe mental disorder. Arch Gen Psychiatry. 2009; 66:713-20.

94. Vancampfort D, Mitchell AJ, De Hert M, Sienaert P, Probst M, Buys R, Stubbs B. Prevalence and predictors of type 2 diabetes mellitus in people with bipolar disorder: a systematic review and meta-analysis. J Clin Psychiatry. 2015;76:1490-9.

95. Vancampfort D, Mitchell AJ, De Hert M, Sienaert P, Probst M, Buys R, Stubbs B. Type 2 diabetes in patients with major depressive disorder: a metaanalysis of prevalence estimates and predictors. Depression Anxiety. 2015; 32:763-73.

96. Yu M, Zhang X, Lu F, Fang L. Depression and risk for diabetes: a Metaanalysis. Can J Diabetes. 2015;39:266-72.

97. Petersen I, Lund C, Stein DJ. Optimizing mental health services in lowincome and middle-income countries. Curr Opin Psychiatry. 2011;24:318-23.

98. Toftegaard KL, Gustafsson LN, Uwakwe R, Andersen UA, Becker T, Bickel GG, Bork B, Cordes J, Frasch K, Jacobsen BA, et al. Where are patients who have co-occurring mental and physical diseases located? Int I Soc Psychiatry. 2015:61:456-64

99. Chereches RM, Litan CM, Zlati AM, Bloom JR. Does co-morbid depression impact diabetes related costs? Evidence from a cross-sectional survey in a low-income country. J Ment Health Policy Econ. 2012;15:127-38.

100. Solano JP, Gomes B, Higginson IJ. A comparison of symptom prevalence in far advanced cancer, AIDS, heart disease, chronic obstructive pulmonary disease and renal disease. J Pain Symptom Manag. 2006;31:58-69.

101. Ren Y, Yang H, Browning C, Thomas S, Liu M. Prevalence of depression in coronary heart disease in China: a systematic review and meta-analysis. Chin Med J. 2014;127:2991-8.

102. Abou Abbas L, Salameh P, Nasser W, Nasser Z, Godin I. Obesity and symptoms of depression among adults in selected countries of the Middle East: a systematic review and meta-analysis. Clin Obes. 2015;5:2-11.

103. Luppino FS, de Wit LM, Bouvy PF, Stijnen T, Cuijpers P, Penninx BWJH, Zitman FG. Overweight, obesity, and depression: a systematic review and meta-analysis of longitudinal studies. Arch Gen Psychiatry. 2010;67:220-9.

104. Mannan M, Mamun A, Doi S, Clavarino A. Is there a bi-directional relationship between depression and obesity among adult men and women? Systematic review and bias-adjusted meta analysis. Asian J Psychiatr. 2016;21:51-66

105. Gariepy G, Nitka D, Schmitz N. The association between obesity and anxiety disorders in the population: a systematic review and meta-analysis. Int J Obes (Lond). 2010;34:407-19.

106. Prince M, Patel V, Saxena S, Maj M, Maselko J, Phillips MR, Rahman A. No health without mental health. Lancet. 2007;370:859-77.

Ready to submit your research? Choose BMC and benefit from:

- fast, convenient online submission

- thorough peer review by experienced researchers in your field

- rapid publication on acceptance

- support for research data, including large and complex data types

- gold Open Access which fosters wider collaboration and increased citations

- maximum visibility for your research: over $100 \mathrm{M}$ website views per year

At BMC, research is always in progress.

Learn more biomedcentral.com/submissions 